\title{
NECESSARY AND SUFFICIENT OPTIMALITY CONDITIONS FOR ELLIPTIC CONTROL PROBLEMS WITH FINITELY MANY POINTWISE STATE CONSTRAINTS*
}

\author{
EDUARDO CASAS ${ }^{1}$
}

\begin{abstract}
The goal of this paper is to prove the first and second order optimality conditions for some control problems governed by semilinear elliptic equations with pointwise control constraints and finitely many equality and inequality pointwise state constraints. To carry out the analysis we formulate a regularity assumption which is equivalent to the first order optimality conditions. Though the presence of pointwise state constraints leads to a discontinuous adjoint state, we prove that the optimal control is Lipschitz in the whole domain. Necessary and sufficient second order conditions are proved with a minimal gap between them.
\end{abstract}

Mathematics Subject Classification. 49K20, 35J25.

Received May 3, 2006. Revised November 20, 2006.

Published online December 21, 2007.

\section{Introduction}

In this paper we study an optimal control problem governed by a semilinear elliptic equation. Bound constraints on the control and finitely many equality and inequality pointwise state constraints are included in the formulation of the problem. The aim is to derive the necessary and sufficient first and second order conditions for a local minimum, as well as to prove that these local minima are Lipschitz functions. The last property is crucial to derive the error estimates in the numerical approximation of the control problem; see, for instance, [1] and [4]. The sufficient second order optimality conditions are also required to derive these error estimates. The necessary second order optimality conditions are not useful in such a context, but they are the reference that should guide the statement for a sufficient second order condition for optimality. In this sense, it is well known that there is a gap between the necessary and sufficient conditions, but in our formulation the gap will be minimal.

As in any optimization problem submitted to some nonlinear constraints, we need a regularity assumption to get the first and second order necessary optimality conditions, the classical assumption is the linear independence of the gradients of the active constraints, which allows to prove the well known Kuhn-Tucker first order optimality conditions. This regularity assumption is also used to prove the second order necessary conditions, however it

Keywords and phrases. Elliptic control problems, pointwise state constraints, Pontryagin's principle, second order optimality conditions.

* This research was partially supported by Ministerio de Educación y Ciencia (Spain).

1 Dpt. Matemática Aplicada y Ciencias de la Computación, E.T.S.I.I y T., Universidad de Cantabria, Av. Los Castros s/n 39005 Santander, Spain; eduardo.casas@unican.es 
is not needed to prove the sufficient second order conditions for optimality. We will follow the same steps for our control problem. Our situation is more complicated because we have infinitely many control constraints (the bound constraints), so we need to formulate a regularity assumption for the state constraints compatible with these control constraints. This is done in (3.1). This regularity assumption was first formulated by Casas and Tröltzsch [7,8]; see also Casas [4] and Casas and Mateos [5]. However in these papers the state constraints were of integral type, so that the pointwise constraints were not included. Here we will prove that the regularity assumption (3.1) is the correct one, in the sense that whenever the first order optimality conditions hold, then this assumption is fulfilled. This is a quite surprising result, it says that the first order optimality conditions are fulfilled if and only if (3.1) holds. As a consequence we have that (3.1) is equivalent to the well known Robinson's regularity condition [18], just for the control problem studied in this paper.

As far as we know there is only one paper dealing with the second-order optimality conditions for pointwise state constrained control problems governed by elliptic partial differential equations; see Casas, Tröltzsch and Unger [10]. Here we consider a particular case of [10], but we improve the results given there in the sense that we prove not only sufficient, but also necessary optimality conditions, with a minimal gap. Indeed the cone of critical directions used in the formulation of the sufficient second-order optimality conditions of [10] is much bigger than the one used in this paper. The parabolic case was considered by Raymond and Tröltzsch [17]. Both papers [10] and [17] rely on the ideas by Maurer and Zowe [16]. In the present paper we use not only the Lagrangian function to write the second order optimality conditions, but also the Hamiltonian, which leads to a better result. The reader is also referred to Casas and Mateos [5] for the use of both functionals in the optimality conditions.

From the first order conditions we deduce that the local minima are Lipschitz functions in the whole domain. This is a surprising property because the adjoint states are discontinuous functions due to the presence of Dirac measures in the adjoint state equation, motivated by the pointwise constraints. Nevertheless we will see that the presence of control constraints along with the well known behavior of Green's functions around the singularities lead to the Lipschitz regularity of the optimal controls.

The plan of the paper is as follows. In Section 2 the control problem is formulated, then the existence of solutions and the differentiability properties of the functions involved in the problem are stated. In Section 3 the regularity assumption is given, then we derive the first order optimality conditions and we deduce the regularity of the optimal control. Finally in Section 4 we prove the necessary and sufficient second order optimality conditions.

In a forthcoming paper the analysis carried out in the present paper will be used to derive some error estimates in the numerical approximation of the control problem by using finite elements.

\section{The CONTROL PROBLEM}

Let $\Omega$ be an open bounded set in $\mathbb{R}^{n}(n=2$ or 3$), \Gamma$ its boundary of class $C^{1,1}$ and $A$ an elliptic operator of the form

$$
A y=-\sum_{i, j=1}^{N} \partial_{x_{j}}\left[a_{i j} \partial_{x_{i}} y\right]+a_{0} y,
$$

where the coefficients $a_{i j}$ belong to $C^{0,1}(\bar{\Omega})$ and satisfy

$$
\lambda_{A}\|\xi\|^{2} \leq \sum_{i, j=1}^{n} a_{i j}(x) \xi_{i} \xi_{j} \quad \forall \xi \in \mathbb{R}^{n} \text { and } \forall x \in \Omega
$$

for some $\lambda_{A}>0$. We also assume that $a_{0} \in L^{\infty}(\Omega)$ and $a_{0}(x) \geq 0$. Let $f: \Omega \times \mathbb{R} \rightarrow \mathbb{R}$ and $L: \Omega \times \mathbb{R}^{2} \longrightarrow \mathbb{R}$ be Carathèodory functions. Given a finite set of points $\left\{x_{j}\right\}_{j=1}^{n_{e}+n_{i}} \subset \Omega$ and real numbers $\left\{\sigma_{j}\right\}_{j=1}^{n_{e}+n_{i}}$, the control 
problem is formulated as follows

$$
(\mathrm{P})\left\{\begin{array}{l}
\min J(u)=\int_{\Omega} L\left(x, y_{u}(x), u(x)\right) \mathrm{d} x \\
\text { subject to }\left(y_{u}, u\right) \in\left(C(\bar{\Omega}) \cap H^{1}(\Omega)\right) \times L^{\infty}(\Omega) \\
\alpha(x) \leq u(x) \leq \beta(x) \quad \text { a.e. } x \in \Omega \\
y_{u}\left(x_{j}\right)=\sigma_{j}, \quad 1 \leq j \leq n_{e} \\
y_{u}\left(x_{j}\right) \leq \sigma_{j}, \quad n_{e}+1 \leq j \leq n_{e}+n_{i}
\end{array}\right.
$$

where $-\infty<\alpha(x)<\beta(x)<+\infty$ for every $x \in \bar{\Omega}, \alpha, \beta \in C^{0,1}(\bar{\Omega})$, and $y_{u}$ is the solution of the state equation

$$
\left\{\begin{array}{l}
A y_{u}+f\left(\cdot, y_{u}\right)=u \text { in } \Omega, \\
y_{u}=0 \text { on } \Gamma
\end{array}\right.
$$

Let us state the assumptions on the functionals $L$ and $f$.

(A1) $f$ is of class $C^{2}$ with respect to the second variable,

$$
f(\cdot, 0) \in L^{p}(\Omega), \quad \frac{\partial f}{\partial y}(x, y) \geq 0
$$

for a fixed $p>n$, and for all $M>0$ there exists a constant $C_{f, M}>0$ such that

$$
\begin{gathered}
\left|\frac{\partial f}{\partial y}(x, y)\right|+\left|\frac{\partial^{2} f}{\partial y^{2}}(x, y)\right| \leq C_{f, M} \text { for a.e. } x \in \Omega \text { and }|y| \leq M \\
\left|\frac{\partial^{2} f}{\partial y^{2}}\left(x, y_{2}\right)-\frac{\partial^{2} f}{\partial y^{2}}\left(x, y_{1}\right)\right|<C_{f, M}\left|y_{2}-y_{1}\right| \text { for }\left|y_{1}\right|,\left|y_{2}\right| \leq M \text { and } x \in \Omega .
\end{gathered}
$$

(A2) $L: \Omega \times \mathbb{R} \times \mathbb{R} \longrightarrow \mathbb{R}$ is of class $C^{2}$ with respect to the second and third variables, $L(\cdot, 0,0) \in L^{1}(\Omega)$, and for all $M>0$ there exist a constant $C_{L, M}>0$ and a function $\psi_{M} \in L^{p}(\Omega)$ (with $p>n$ given in (A1)) such that

$$
\begin{gathered}
\left|\frac{\partial L}{\partial y}(x, y, u)\right| \leq \psi_{M}(x), \quad\left\|D_{(y, u)}^{2} L(x, y, u)\right\| \leq C_{L, M}, \\
\left|\frac{\partial L}{\partial u}\left(x_{2}, y, u\right)-\frac{\partial L}{\partial u}\left(x_{1}, y, u\right)\right| \leq C_{L, M}\left|x_{2}-x_{1}\right|, \\
\left\|D_{(y, u)}^{2} L\left(x, y_{2}, u_{2}\right)-D_{(y, u)}^{2} L\left(x, y_{1}, u_{1}\right)\right\| \leq C_{L, M}\left(\left|y_{2}-y_{1}\right|+\left|u_{2}-u_{1}\right|\right),
\end{gathered}
$$

for a.e. $x, x_{i} \in \Omega$ and $|y|,\left|y_{i}\right|,|u|,\left|u_{i}\right| \leq M, i=1,2$, where $D_{(y, u)}^{2} L$ denotes the second derivative of $L$ with respect to $(y, u)$.

Now we have the following result about the existence of a solution for (2.1) as well as for the problem (P).

Theorem 2.1. Suppose (A1) holds. Then for every $u \in L^{r}(\Omega), 2 \leq r \leq p$, the state equation (2.1) has a unique solution $y_{u}$ in the space $W^{2, r}(\Omega)$. Furthermore if the function $L$ is convex with respect to the third component, (A2) holds and the set of feasible controls is nonempty, then the control problem has at least one solution.

The existence of a unique solution of $(2.1)$ in $H^{1}(\Omega) \cap L^{\infty}(\Omega)$ is classical and it is a consequence of the monotonicity of $f$ with respect to the second component. The $W^{2, r}(\Omega)$ regularity can be got from the results by Grisvard [12]. The bound $r \leq p$ is a consequence of the hypothesis $f(\cdot, 0) \in L^{p}(\Omega)$ (see assumption (A1)). The existence of a solution of $(\mathrm{P})$ follows from the convexity of $L$ with respect to $u$; see Casas and Mateos [6] for the proof.

We finish this section by recalling some results about the differentiability of the functionals involved in the control problem. For the detailed proofs the reader is referred to Casas and Mateos [5]. 
Theorem 2.2. If (A1) holds, then the mapping $G: L^{r}(\Omega) \longrightarrow W^{2, r}(\Omega)(2 \leq r \leq p)$, defined by $G(u)=y_{u}$ is of class $C^{2}$. Moreover for all $v, u \in L^{r}(\Omega), z_{v}=G^{\prime}(u) v$ is the solution of

$$
\left\{\begin{array}{l}
A z_{v}+\frac{\partial f}{\partial y}\left(x, y_{u}\right) z_{v}=v \text { in } \Omega \\
z_{v}=0 \text { on } \Gamma
\end{array}\right.
$$

Finally, for every $v_{1}, v_{2} \in L^{r}(\Omega), z_{v_{1} v_{2}}=G^{\prime \prime}(u) v_{1} v_{2}$ is the solution of

$$
\left\{\begin{aligned}
A z_{v_{1} v_{2}}+\frac{\partial f}{\partial y}\left(x, y_{u}\right) z_{v_{1} v_{2}}+\frac{\partial^{2} f}{\partial y^{2}}\left(x, y_{u}\right) z_{v_{1}} z_{v_{2}} & =0 \text { in } \Omega \\
z_{v_{1} v_{2}} & =0 \text { on } \Gamma,
\end{aligned}\right.
$$

where $z_{v_{i}}=G^{\prime}(u) v_{i}, i=1,2$.

The proof can be obtained by using the implicit function theorem.

Theorem 2.3. Let us suppose that (A1) and (A2) hold. Then the functional $J: L^{\infty}(\Omega) \rightarrow \mathbb{R}$ is of class $C^{2}$. Moreover, for every $u, v, v_{1}, v_{2} \in L^{\infty}(\Omega)$

$$
J^{\prime}(u) v=\int_{\Omega}\left(\frac{\partial L}{\partial u}\left(x, y_{u}, u\right)+\varphi_{0 u}\right) v \mathrm{~d} x
$$

and

$$
\begin{aligned}
J^{\prime \prime}(u) v_{1} v_{2}= & \int_{\Omega}\left[\frac{\partial^{2} L}{\partial y^{2}}\left(x, y_{u}, u\right) z_{v_{1}} z_{v_{2}}+\frac{\partial^{2} L}{\partial y \partial u}\left(x, y_{u}, u\right)\left(z_{v_{1}} v_{2}+z_{v_{2}} v_{1}\right)\right. \\
& \left.+\frac{\partial^{2} L}{\partial u^{2}}\left(x, y_{u}, u\right) v_{1} v_{2}-\varphi_{0 u} \frac{\partial^{2} f}{\partial y^{2}}\left(x, y_{u}\right) z_{v_{1}} z_{v_{2}}\right] \mathrm{d} x
\end{aligned}
$$

where $y_{u}=G(u)$ and $\varphi_{0 u} \in W^{2, p}(\Omega)$ is the unique solution of the problem

$$
\left\{\begin{array}{l}
A^{*} \varphi+\frac{\partial f}{\partial y}\left(x, y_{u}\right) \varphi=\frac{\partial L}{\partial y}\left(x, y_{u}, u\right) \text { in } \Omega \\
\varphi=0 \text { on } \Gamma
\end{array}\right.
$$

$A^{*}$ being the adjoint operator of $A$ and $z_{v_{i}}=G^{\prime}(u) v_{i}, i=1,2$.

Theorem 2.4. Let us suppose that (A1) holds. Then for each $j$, the functional $G_{j}: L^{r}(\Omega) \rightarrow \mathbb{R}(2 \leq r \leq p)$, defined by $G_{j}(u)=y_{u}\left(x_{j}\right)-\sigma_{j}$, is of class $C^{2}$. Moreover, for every $u, v, v_{1}, v_{2} \in L^{r}(\Omega)$

$$
G_{j}^{\prime}(u) v=z_{v}\left(x_{j}\right)=\int_{\Omega} \varphi_{j u} v \mathrm{~d} x
$$

and

$$
G_{j}^{\prime \prime}(u) v_{1} v_{2}=-\int_{\Omega} \varphi_{j u} \frac{\partial^{2} f}{\partial y^{2}}\left(x, y_{u}\right) z_{v_{1}} z_{v_{2}} \mathrm{~d} x
$$

where $y_{u}=G(u), z_{v} \in W^{2, r}(\Omega)$ is the solution of $(2.2), \varphi_{j u} \in W^{1, s}(\Omega)$, for any $1 \leq s<n /(n-1)$, is the unique solution of the problem

$$
\left\{\begin{array}{l}
A^{*} \varphi+\frac{\partial f}{\partial y}\left(x, y_{u}\right) \varphi=\delta_{x_{j}} \text { in } \Omega \\
\varphi=0 \text { on } \Gamma
\end{array}\right.
$$

and $z_{v_{i}}=G^{\prime}(u) v_{i}, i=1,2$.

The last two theorems follow from Theorem 2.2 and the chain rule. 


\section{First order optimality CONDITIONS}

We start this section by reformulating problem (P) with the help of the functionals $G_{j}$ introduced in Theorem 2.4.

$$
(\mathrm{P})\left\{\begin{array}{l}
\text { Minimize } J(u), \\
\alpha(x) \leq u(x) \leq \beta(x) \text { a.e. } x \in \Omega, \\
G_{j}(u)=0,1 \leq j \leq n_{e}, \\
G_{j}(u) \leq 0, n_{e}+1 \leq j \leq n_{e}+n_{i} .
\end{array}\right.
$$

This is a more convenient form to derive the first order optimality conditions. Let us introduce some notation. Fixed a feasible control $\bar{u}$ and given $\varepsilon>0$, we denote the set of $\varepsilon$-inactive constraints by

$$
\Omega_{\varepsilon}=\{x \in \Omega: \alpha(x)+\varepsilon<\bar{u}(x)<\beta(x)-\varepsilon\} .
$$

We say that a feasible control $\bar{u}$ is regular if the following assumption is fulfilled

$$
\left\{\begin{array}{l}
\exists \varepsilon_{\bar{u}}>0 \text { and }\left\{\bar{w}_{j}\right\}_{j \in I_{0}} \subset L^{\infty}(\Omega), \text { with supp } \bar{w}_{j} \subset \Omega_{\varepsilon_{\bar{u}}}, \text { such that } \\
G_{i}^{\prime}(\bar{u}) \bar{w}_{j}=\delta_{i j}, \quad i, j \in I_{0}
\end{array}\right.
$$

where

$$
I_{0}=\left\{j \leq n_{e}+n_{i} \mid G_{j}(\bar{u})=0\right\} .
$$

$I_{0}$ is the set of indices corresponding to active constraints. The question we now set up is whether this regularity assumption is realistic or not. A first answer is given by the following theorem:

Theorem 3.1. If $\bar{u}$ is a feasible control for problem $(\mathrm{P})$ and there exists $\varepsilon>0$ such that $\Omega_{\varepsilon}$ has a nonempty interior, then (3.1) holds. In particular if $\bar{u}$ is continuous and is not identically equal to $\alpha$ or $\beta$, then (3.1) holds.

Proof. Let us assume that $\varepsilon>0$ and $\Omega_{\varepsilon}$ has a nonempty interior. Let $B \subset \Omega_{\varepsilon}$ be any open ball and let us define $\mathcal{S}: L^{2}(B) \longrightarrow \mathbb{R}^{\left|I_{0}\right|}$ by

$$
\mathcal{S} v=\left(G_{j}^{\prime}(\bar{u}) v\right)_{j \in I_{0}}=\left(z_{v}\left(x_{j}\right)\right)_{j \in I_{0}}=\left(\int_{\Omega} \varphi_{j \bar{u}} v \mathrm{~d} x\right)_{j \in I_{0}}
$$

where $v$ is extended to $\Omega$ by zero, $z_{v} \in H^{2}(\Omega)$ is the solution of $(2.2)$ and $\left|I_{0}\right|$ denotes the cardinal of $I_{0}$. If $\mathcal{S}$ is surjective, then we deduce the existence of functions $\left\{\bar{w}_{j}\right\}_{j \in I_{0}}$, with $\operatorname{supp} \bar{w}_{j} \subset B$, satisfying (3.1), which proves the theorem. Let us argue by contradiction and suppose that $\mathcal{S}$ is not surjective, then there exists a nonzero vector $\xi \in \mathbb{R}^{\left|I_{0}\right|}$ such that

$$
\sum_{j \in I_{0}} \xi_{j} z_{v}\left(x_{j}\right)=\xi^{T} \mathcal{S} v=0 \quad \forall v \in L^{2}(B) .
$$

Let us take $\varphi \in W^{1, s}(\Omega), 1 \leq s<n /(n-1)$, satisfying

$$
\left\{\begin{array}{l}
A^{*} \varphi+\frac{\partial f}{\partial y}\left(x, y_{\bar{u}}\right) \varphi=\sum_{j \in I_{0}} \xi_{j} \delta_{x_{j}} \text { in } \Omega \\
\varphi=0 \text { on } \Gamma .
\end{array}\right.
$$

Doing an integration by parts and using (2.2) we get

$$
\int_{B} \varphi(x) v(x) \mathrm{d} x=\int_{\Omega}\left(A z_{v}+\frac{\partial f}{\partial y}\left(x, y_{\bar{u}}(x) z_{v}\right)\right) \varphi \mathrm{d} x=\sum_{j \in I_{0}} \xi_{j}\left\langle\delta_{x_{j}}, z_{v}\right\rangle=\sum_{j \in I_{0}} \xi_{j} z_{v}\left(x_{j}\right)=0 \quad \forall v \in L^{2}(B) .
$$


Therefore $\varphi=0$ in $B$ and

$$
A^{*} \varphi+\frac{\partial f}{\partial y}\left(x, y_{\bar{u}}\right) \varphi=0 \text { in } \Omega \backslash\left\{x_{j}\right\}_{j \in I_{0}} .
$$

This implies that $\varphi=0$ in $\Omega \backslash\left\{x_{j}\right\}_{j \in I_{0}}$ and also in $\Omega$; see, for instance, Saut and Scheurer [19]. Then the right hand side of the equation satisfied by $\varphi$ must be zero and therefore $\xi=0$, which is a contradiction with the choice of $\xi$.

Corollary 3.1. Let $\bar{u}$ be a feasible control for problem $(\mathrm{P})$ and let us assume that $\Omega_{\varepsilon}$ has a nonempty interior. Then the functions $\left\{\bar{w}_{j}\right\}_{j \in I_{0}}$ verifying (3.1) can be chosen of class $C^{\infty}$ in $\Omega$ and support in any given ball $B \subset \Omega_{\varepsilon}$.

Proof. It is enough to remark that $B$ was taken in the proof of Theorem 2.1 as any ball contained in $\bar{\Omega}_{\varepsilon}$. On the other hand, since the functions of class $C^{\infty}$ and support contained in $B$ are dense in $L^{2}(B)$, then $\mathcal{S}$ is also surjective from this class of regular functions to $\mathbb{R}^{\left|I_{0}\right|}$, which proves the corollary.

Let us write now the first order optimality conditions. Associated with problem $(\mathrm{P})$ we consider the Lagrangian function $\mathcal{L}: L^{\infty}(\Omega) \times \mathbb{R}^{n_{e}+n_{i}} \longrightarrow \mathbb{R}$ given by

$$
\mathcal{L}(u, \lambda)=J(u)+\sum_{j=1}^{n_{e}+n_{i}} \lambda_{j} G_{j}(u)=J(u)+\sum_{j=1}^{n_{e}+n_{i}} \lambda_{j}\left(y_{u}\left(x_{j}\right)-\sigma_{j}\right) .
$$

Under the regularity assumption (3.1) we can derive the first order necessary conditions for optimality in a qualified form. For the proof the reader is referred to Bonnans and Casas [2] or Clarke [11]; see also Mateos [15].

Theorem 3.2. Let us assume that $\bar{u}$ is a local solution of $(\mathrm{P})$ and (3.1) holds. Then there exist real numbers $\left\{\bar{\lambda}_{j}\right\}_{j=1}^{n_{e}+n_{i}}$ such that

$$
\begin{gathered}
\bar{\lambda}_{j} \geq 0 \quad \text { and } \bar{\lambda}_{j} G_{j}(\bar{u})=0, \quad \text { if } n_{e}+1 \leq j \leq n_{e}+n_{i} \\
\frac{\partial \mathcal{L}}{\partial u}(\bar{u}, \bar{\lambda})(u-\bar{u}) \geq 0 \quad \text { for all } \alpha \leq u \leq \beta
\end{gathered}
$$

Denoting by $\bar{\varphi}_{0}$ and $\bar{\varphi}_{j}$ the solutions of (2.6) and (2.9) corresponding to $\bar{u}$ and setting

$$
\bar{\varphi}=\bar{\varphi}_{0}+\sum_{j=1}^{n_{e}+n_{i}} \bar{\lambda}_{j} \bar{\varphi}_{j}
$$

we deduce from Theorems 2.3 and 2.4 and the definition of $\mathcal{L}$ that

$$
\begin{aligned}
\frac{\partial \mathcal{L}}{\partial u}(\bar{u}, \bar{\lambda}) v & =\int_{\Omega}\left(\frac{\partial L}{\partial u}(x, \bar{y}, \bar{u})+\bar{\varphi}_{0}\right) v \mathrm{~d} x+\sum_{j=1}^{n_{e}+n_{i}} \bar{\lambda}_{j} \int_{\Omega} \bar{\varphi}_{j} v \mathrm{~d} x \\
& =\int_{\Omega}\left(\frac{\partial L}{\partial u}(x, \bar{y}, \bar{u})+\bar{\varphi}\right) v \mathrm{~d} x=\int_{\Omega} \bar{d}(x) v(x) \quad \forall v \in L^{2}(\Omega),
\end{aligned}
$$

where $\bar{y}=G(\bar{u})=y_{\bar{u}}$ and

$$
\bar{d}(x)=\frac{\partial L}{\partial u}(x, \bar{y}(x), \bar{u}(x))+\bar{\varphi}(x) .
$$

From (3.3) we deduce that

$$
\bar{d}(x)=\left\{\begin{array}{cl}
0 & \text { for a.e. } x \in \Omega \text { where } \alpha(x)<\bar{u}(x)<\beta(x), \\
\geq 0 & \text { for a.e. } x \in \Omega \text { where } \bar{u}(x)=\alpha(x) \\
\leq 0 & \text { for a.e. } x \in \Omega \text { where } \bar{u}(x)=\beta(x) .
\end{array}\right.
$$


The following theorem states the uniqueness of the Lagrange multipliers.

Theorem 3.3. Under the assumptions of Theorem 3.2, the Lagrange multipliers $\left\{\bar{\lambda}_{j}\right\}_{j=1}^{n_{e}+n_{i}}$ are unique and they are given by the expressions

$$
\bar{\lambda}_{j}=\left\{\begin{array}{l}
-\int_{\Omega}\left(\frac{\partial L}{\partial u}(x, \bar{y}(x), \bar{u}(x))+\bar{\varphi}_{0}(x)\right) \bar{w}_{j}(x) \mathrm{d} x \text { if } j \in I_{0} \\
0 \text { otherwise, }
\end{array}\right.
$$

where $\left\{\bar{w}_{j}\right\}_{j \in I_{0}}$ are introduced in (3.1).

Proof. Let $j \in I_{0}$. Since supp $\bar{w}_{j} \subset \Omega_{\varepsilon_{\bar{u}}}$, if we take $\rho \in \mathbb{R}$ satisfying

$$
|\rho|<\frac{\varepsilon_{\bar{u}}}{\left\|\bar{w}_{j}\right\|_{L^{\infty}(\Omega)}},
$$

we get

$$
\alpha(x) \leq u_{\rho}(x)=\bar{u}(x)+\rho \bar{w}_{j}(x) \leq \beta(x) \text { a.e. } x \in \Omega .
$$

Taking $u=u_{\rho}$ in (3.3) with $\rho$ positive and negative respectively, we deduce

$$
\int_{\Omega}\left(\frac{\partial L}{\partial u}(x, \bar{y}(x), \bar{u}(x))+\bar{\varphi}_{0}(x)\right) \bar{w}_{j}(x) \mathrm{d} x+\bar{\lambda}_{j}=\frac{\partial \mathcal{L}}{\partial u}(\bar{u}, \bar{\lambda}) \bar{w}_{j}=0 .
$$

If $j \notin I_{0}$, then (3.2) implies that $\bar{\lambda}_{j}=0$.

From the first order optimality condition we deduce the regularity of the local minima.

Theorem 3.4. Suppose that $\bar{u}$ be a feasible control of (P) satisfying (3.2)-(3.3). Moreover let us assume that there exists $\lambda_{L}>0$ such that

$$
\frac{\partial^{2} L}{\partial u^{2}}(x, y, u) \geq \lambda_{L}, \text { a.e. } x \in \Omega \text { and }(y, u) \in \mathbb{R}^{2} .
$$

Then, for all $x \in \bar{\Omega} \backslash\left\{x_{j}\right\}_{j \in I_{\bar{u}}}$, the equation

$$
\bar{\varphi}(x)+\frac{\partial L}{\partial u}(x, \bar{y}(x), t)=0,
$$

has a unique solution $\bar{t}=\bar{s}(x)$, where

$$
I_{\bar{u}}=\left\{j \in I_{0}: \bar{\lambda}_{j} \neq 0\right\}
$$

The mapping $\bar{s}$ belongs to $C_{\text {loc }}^{0,1}\left(\bar{\Omega} \backslash\left\{x_{j}\right\}_{j \in I_{\bar{u}}}\right)$. Moreover $\bar{u}$ and $\bar{s}$ are related by the formula

$$
\bar{u}(x)=\operatorname{Proj}_{[\alpha(x), \beta(x)]}(\bar{s}(x))=\max (\alpha(x), \min (\beta(x), \bar{s}(x))),
$$

and $\bar{u} \in C^{0,1}(\bar{\Omega})$.

Proof. From (3.4), (2.6) and (2.9) we deduce that $\bar{\varphi}_{0} \in W^{2, p}(\Omega) \subset C^{1}(\bar{\Omega})$ and $\bar{\varphi}_{j} \in W_{l o c}^{2, p}\left(\Omega \backslash\left\{x_{j}\right\}\right) \subset C^{1}(\bar{\Omega} \backslash$ $\left.\left\{x_{j}\right\}\right), 1 \leq j \leq n_{e}+n_{i}$. Furthermore it is well known, see for instance [14], that the asymptotic behavior of $\bar{\varphi}_{j}(x)$ when $x \rightarrow x_{j}$ is of type

$$
\bar{\varphi}_{j}(x) \approx \begin{cases}\left(C_{1} \log \frac{1}{\left|x-x_{j}\right|}+C_{2}\right) & \text { if } n=2 \\ \left(C_{1} \frac{1}{\left|x-x_{j}\right|^{n-2}}+C_{2}\right) & \text { if } n>2\end{cases}
$$


with $C_{1}>0$. In particular we have that $\bar{\varphi} \in C_{l o c}^{0,1}\left(\bar{\Omega} \backslash\left\{x_{j}\right\}_{j \in I_{\bar{u}}}\right)$. Now arguing as in [1], Lemma 3.1, we deduce that (3.9) has a unique solution for every $x \in \bar{\Omega} \backslash\left\{x_{j}\right\}_{j \in I_{\bar{u}}}, \bar{s} \in C_{\text {loc }}^{0,1}\left(\bar{\Omega} \backslash\left\{x_{j}\right\}_{j \in I_{\bar{u}}}\right)$ and (3.10) holds. Now taking into account (3.11), we deduce that for every $M>0$ there exists $\varepsilon_{M}>0$ such that

$$
|\bar{d}(x)|>M \quad \forall x \in \bigcup_{j \in I_{\bar{u}}} B_{\varepsilon_{M}}\left(x_{j}\right)
$$

the sign of $\bar{d}(x)$ in $B_{\varepsilon_{M}}\left(x_{j}\right)$ being equal to the sign of the Lagrange multiplier $\bar{\lambda}_{j}$. Taking $M$ large enough, from (3.6) we deduce

$$
\bar{u}(x)=\left\{\begin{array}{ll}
\alpha(x) & \text { if } \bar{\lambda}_{j}>0 \\
\beta(x) & \text { if } \bar{\lambda}_{j}<0
\end{array} \quad \forall x \in B_{\varepsilon_{M}}\left(x_{j}\right) .\right.
$$

Combining this with (3.10), the regularity of $\bar{s}$ and the fact that $\alpha, \beta \in C^{0,1}(\bar{\Omega})$, then we get $\bar{u} \in C^{0,1}(\bar{\Omega})$.

Remark 3.1. The reader should remark that the previous theorem is obvious in the very frequent case where $L(x, y, u)=\left[\left(y-y_{d}(x)\right)^{2}+N u^{2}\right] / 2$, with $N>0$. In this case equation (3.9) is written in the way $\bar{\varphi}(x)+N t=0$, then $\bar{s}(x)=-\bar{\varphi}(x) / N$ and

$$
\bar{u}(x)=\operatorname{Proj}_{[\alpha(x), \beta(x)]}\left(-\frac{1}{N} \bar{\varphi}(x)\right) .
$$

In particular, it is important to remark that the assumption $N>0$, equivalent to (3.8) for this cost functional, is essential to derive the regularity of $\bar{u}$. In the case $N=0$, the behavior of $\bar{u}$ is of Bang-Bang type.

Corollary 3.2. Under the assumptions of Theorem 3.4, the control $\bar{u}$ satisfies the regularity assumption (3.1).

This corollary is an immediate consequence of Theorems 3.1 and 3.4. It states, under the extra assumption (3.8), the existence of Lagrange multipliers for a local minimum of $(\mathrm{P})$ if and only if the regularity assumption (3.1) is fulfilled.

\section{SECOND ORDER OPTIMALITy CONDItions}

Associated with function $\bar{d}$ given by (3.5) we define the set

$$
\Omega^{0}=\{x \in \Omega:|\bar{d}(x)|>0\}
$$

Let $\bar{u}$ be a feasible control for problem (P) satisfying the first order optimality conditions (3.2)-(3.3). We define the cone of critical directions

$$
C_{\bar{u}}^{0}=\left\{h \in L^{2}(\Omega) \text { satisfying (4.3) and } h(x)=0 \text { for a.e. } x \in \Omega^{0}\right\}
$$

with

$$
\left\{\begin{array}{l}
G_{j}^{\prime}(\bar{u}) h=0 \text { if }\left(j \leq n_{e}\right) \text { or }\left(j>n_{e}, G_{j}(\bar{u})=0 \text { and } \bar{\lambda}_{j}>0\right) \\
G_{j}^{\prime}(\bar{u}) h \leq 0 \text { if }\left(j>n_{e}, G_{j}(\bar{u})=0 \text { and } \bar{\lambda}_{j}=0\right) \\
h(x)= \begin{cases}\geq 0 & \text { if } \bar{u}(x)=\alpha(x) \\
\leq 0 & \text { if } \bar{u}(x)=\beta(x) .\end{cases}
\end{array}\right.
$$

Now we are ready to state the second order necessary optimality conditions. 
Theorem 4.1. Let us assume that $\bar{u}$ is a local solution of $(\mathrm{P}),(3.1)$ holds and $\left\{\bar{\lambda}_{j}\right\}_{j=1}^{m}$ are the Lagrange multipliers satisfying (3.2) and (3.3). Then the following inequalities are satisfied

$$
\begin{array}{cc}
\frac{\partial^{2} L}{\partial u^{2}}(x, \bar{y}(x), \bar{u}(x)) \geq 0 & \text { a.e. } x \in \Omega \backslash \Omega^{0} \\
\frac{\partial^{2} \mathcal{L}}{\partial u^{2}}(\bar{u}, \bar{\lambda}) h^{2} \geq 0 \quad \forall h \in C_{\bar{u}}^{0} .
\end{array}
$$

Proof. Inequality (4.4) can be derived as follows. According to Pontryagin's principle (see Casas [3] or Casas, Raymond and Zidani [9]) we know that

$$
H(x, \bar{y}(x), \bar{u}(x), \bar{\varphi}(x))=\min _{k \in[\alpha(x), \beta(x)]} H(x, \bar{y}(x), k, \bar{\varphi}(x)) \text { a.e. } x \in \Omega,
$$

where $H: \Omega \times \mathbb{R}^{3} \longrightarrow \mathbb{R}$ is the Hamiltonian of $(\mathrm{P})$, defined by

$$
H(x, y, u, \varphi)=L(x, y, u)+\varphi[u-f(x, y)] .
$$

Then the classical results on the optimization in $\mathbb{R}$ lead to

$$
\frac{\partial^{2} L}{\partial u^{2}}(x, \bar{y}(x), \bar{u}(x))=\frac{\partial^{2} H}{\partial u^{2}}(x, \bar{y}(x), \bar{u}(x), \bar{\varphi}(x)) \geq 0
$$

whenever

$$
\bar{d}(x)=\frac{\partial H}{\partial u}(x, \bar{y}(x), \bar{u}(x), \bar{\varphi}(x))=0 \Leftrightarrow x \in \Omega \backslash \Omega^{0},
$$

which implies (4.4).

Let us prove (4.5). We first prove (4.5) for functions $h \in C_{\bar{u}}^{0} \cap L^{\infty}(\Omega)$. In this situation we apply Casas and Tröltzsch [8], Theorem 2.2. It is enough to check the assumptions (A1) and (A2) of such a paper. Assumption (A1) says that $J^{\prime}(\bar{u})$ and $G_{j}^{\prime}(\bar{u})$ must be continuous functionals in $L^{2}(\Omega)$, which is an immediate consequence of Theorems 2.3 and 2.4. Assumption (A2) of [8] says that

$$
\frac{\partial \mathcal{L}}{\partial u^{2}}(\bar{u}, \bar{\lambda}) h_{k}^{2} \rightarrow \frac{\partial \mathcal{L}}{\partial u^{2}}(\bar{u}, \bar{\lambda}) h^{2}
$$

whenever $\left\{h_{k}\right\}_{k=1}^{\infty}$ is bounded in $L^{\infty}(\Omega)$ and $h_{k}(x) \rightarrow h(x)$ a.e. $x \in \Omega$. Again this follows easily from Theorems 2.3 and 2.4 .

Now let us assume that $h \in C_{\bar{u}}^{0}$, but $h \notin L^{\infty}(\Omega)$. For every positive integer $k$ we set $\hat{h}_{k}(x)=\operatorname{Proj}_{[-k,+k]}(h(x))$ for $x \in \Omega$. It is clear that $\left\{\hat{h}_{k}\right\}_{k} \subset L^{\infty}(\Omega),\left|\hat{h}_{k}(x)\right| \leq|h(x)|$ and $\hat{h}_{k}(x) \rightarrow h(x)$ a.e. $x \in \Omega$, therefore $\hat{h}_{k} \rightarrow h$ in $L^{2}(\Omega)$. Let us consider the indices

$$
I_{0}(h)=\left\{j \in I_{0}: G_{j}^{\prime}(\bar{u}) h=0\right\} .
$$

Then we have

$$
\alpha_{k j}=G_{j}^{\prime}(\bar{u}) \hat{h}_{k} \rightarrow G_{j}^{\prime}(\bar{u}) h=0 \quad \forall j \in I_{0}(h) .
$$

Finally we set

$$
h_{k}=\hat{h}_{k}-\sum_{i \in I_{0}(h)} \alpha_{k i} \bar{w}_{i},
$$

where $\left\{\bar{w}_{j}\right\}_{j \in I_{0}}$ are the functions given in (3.1). Thus we have that $h_{k} \rightarrow h$ in $L^{2}(\Omega)$ strongly. Let us check that $h_{k} \in C_{\bar{u}}^{0}$ for every $k$ large enough. If $|\bar{d}(x)|>0$, then (3.6) implies that $\bar{u}(x)=\alpha(x)$ or $\bar{u}(x)=\beta(x)$, therefore $\bar{w}_{j}(x)=0$ for every $j \in I_{0}$, hence $\left|h_{k}(x)\right|=\left|\hat{h}_{k}(x)\right| \leq|h(x)|=0$. So $h_{k}$ vanishes in $\Omega_{0}$. Using once again 
that $\bar{w}_{j}(x)=0$ if $\bar{u}(x)=\alpha(x)$ or $\bar{u}(x)=\beta(x)$, we get that the sign of $h_{k}(x)$ is equal to the sign of $h(x)$ when $\bar{u}(x)$ takes the values $\alpha(x)$ or $\beta(x)$, therefore the third sign condition of (4.3) holds.

If $j \in I_{0}$ and $G_{j}^{\prime}(\bar{u}) h<0$, then the convergence $G_{j}^{\prime}(\bar{u}) h_{k} \rightarrow G_{j}^{\prime}(\bar{u}) h$ implies that $G_{j}^{\prime}(\bar{u}) h_{k}<0$ for $k$ large enough. If $G_{j}^{\prime}(\bar{u}) h=0$, then

$$
G_{j}^{\prime}(\bar{u}) h_{k}=G_{j}^{\prime}(\bar{u}) \hat{h}_{k}-\sum_{i \in I_{0}(h)} \alpha_{k i} G_{j}^{\prime}(\bar{u}) \bar{w}_{i}=G_{j}^{\prime}(\bar{u}) \hat{h}_{k}-\alpha_{k j}=0 .
$$

In any case the relations of (4.3) are fulfilled, therefore $h_{k} \in C_{\bar{u}}^{0} \cap L^{\infty}(\Omega)$ for every $k$ large enough. Then (4.5) holds for every $h_{k}$ and then passing to the limit when $k \rightarrow \infty$, using the expressions obtained for the second derivatives of $L$ and $G_{j}$, we get that $h$ verifies (4.5).

Let us remark that inequality (4.4) holds if $L$ is convex with respect to the third component, which is assumed to prove the existence of a solution for problem $(\mathrm{P})$.

The next theorem states the sufficient conditions for optimality.

Theorem 4.2. Let $\bar{u}$ be an admissible control for problem (P) satisfying (3.2)-(3.3) for some $\bar{\lambda}_{j}, j=1, \ldots, n_{i}+$ $n_{e}$. Let us suppose that there exist $\tau>0$ and $\mu>0$ such that

$$
\begin{aligned}
\frac{\partial^{2} L}{\partial u^{2}}(x, \bar{y}(x), \bar{u}(x)) & \geq \mu \text { a.e. } x \in \Omega \backslash \Omega^{\tau} \\
\frac{\partial^{2} \mathcal{L}}{\partial u^{2}}(\bar{u}, \bar{\lambda}) h^{2} & >0 \text { for all } h \in C_{\bar{u}}^{0} \backslash\{0\},
\end{aligned}
$$

where

$$
\Omega^{\tau}=\{x \in \Omega:|\bar{d}(x)|>\tau\} .
$$

Then there exist $\varepsilon>0$ and $\delta>0$ such that

$$
J(\bar{u})+\frac{\delta}{2}\|u-\bar{u}\|_{L^{2}(\Omega)}^{2} \leq J(u)
$$

for all admissible control $u$ with $\|u-\bar{u}\|_{L^{\infty}(\Omega)} \leq \varepsilon$.

The proof is the same as in Casas and Mateos [5], Theorem 4.3. The reader should note that a more general state equation was studied in [5]. It was written in the way

$$
\left\{\begin{array}{l}
A y_{u}=f\left(x, y_{u}, u\right) \text { in } \Omega, \\
y_{u}=0 \text { on } \Gamma .
\end{array}\right.
$$

For this state equation the Hamiltonian becomes

$$
H(x, y, u, \varphi)=L(x, y, u)+\varphi f(x, y, u)
$$

and the second derivative of the Lagrangian is

$$
\frac{\partial^{2} \mathcal{L}}{\partial u^{2}}(\bar{u}, \bar{\lambda}) h^{2}=\int_{\Omega}\left(\bar{H}_{u u}(x) h^{2}(x)+2 \bar{H}_{u y}(x) z_{h}(x) h(x)+\bar{H}_{y y}(x) z_{h}^{2}(x)\right) \mathrm{d} x,
$$

where

$$
\bar{H}_{u u}(x)=\frac{\partial^{2} H}{\partial u^{2}}(x, \bar{y}(x), \bar{u}(x), \bar{\varphi}(x)),
$$


$\bar{H}_{u y}$ and $\bar{H}_{y y}$ being defined analogously, and $z_{h}$ is the solution of the equation

$$
\left\{\begin{array}{l}
A z_{h}=\frac{\partial f}{\partial y}(x, \bar{y}(x), \bar{u}(x)) z_{h}+\frac{\partial f}{\partial u}(x, \bar{y}(x), \bar{u}(x)) h \text { in } \Omega \\
z_{h}=0 \text { on } \Gamma .
\end{array}\right.
$$

There is one difficulty with this more general state equation. In the second derivative of the Lagrangian function the following term appears

$$
\int_{\Omega} \bar{H}_{u u}(x) h^{2}(x) \mathrm{d} x=\int_{\Omega}\left(\frac{\partial^{2} L}{\partial u^{2}}(x, \bar{y}(x), \bar{u}(x))+\bar{\varphi}(x) \frac{\partial^{2} f}{\partial u^{2}}(x, \bar{y}(x), \bar{u}(x))\right) h^{2}(x) \mathrm{d} x .
$$

In [5] it was possible to handle this integral because there was no pointwise state constraints and $\bar{\varphi}$ was a function belonging to $W^{1, s}(\Omega)$ for some $s>n$, in particular bounded, and also $\bar{H}_{u u}$ was a bounded function in $\Omega$. However in the problem we are studying here, the adjoint state is not bounded because of the Dirac measures appearing on the right hand side of the adjoint state equation. This time we are able to handle the above integral because the control appears linearly in the state equation and then

$$
\bar{H}_{u u}(x)=\frac{\partial^{2} H}{\partial u^{2}}(x, \bar{y}(x), \bar{u}(x), \bar{\varphi}(x))=\frac{\partial^{2} L}{\partial u^{2}}(x, \bar{y}(x), \bar{u}(x))
$$

which is a bounded function. Just by taking into account this fact, the proof of [5], Theorem 4.3, is still valid here.

Remark 4.1. If we assume that $L$ satisfies (3.8), then the necessary and sufficient conditions for optimality of Theorems 4.1 and 4.2 are reduced to (4.5) and (4.7), respectively. Under this assumption we see that the gap between the necessary and sufficient conditions is minimal. On the other hand, we should remark that the regularity hypothesis (3.1) is required to derive the necessary conditions, but it is not needed to prove the sufficiency of the optimality conditions given in Theorem 4.2. This is the same situation that we find in finite dimension.

In order to carry out the numerical analysis of (P), it is usual to do a sufficient second order optimality condition which seems to be a stronger assumption than (4.6)-(4.7), but we are going to see that it is not the case.

Theorem 4.3. Let $\bar{u}$ be an admissible control for problem $(\mathrm{P})$ satisfying the first order optimality conditions (3.2)-(3.3). If (4.6)-(4.7) hold, then there exist $\bar{\mu}>0$ and $\bar{\tau}>0$ such that

$$
\frac{\partial^{2} \mathcal{L}}{\partial u^{2}}(\bar{u}, \bar{\lambda}) h^{2} \geq \bar{\mu}\|h\|_{L^{2}(\Omega)}^{2} \text { for all } h \in C_{\bar{u}}^{\bar{\tau}}
$$

where

$$
C_{\bar{u}}^{\bar{\tau}}=\left\{h \in L^{2}(\Omega) \text { satisfying (4.3) and } h(x)=0 \text { a.e. } x \in \Omega^{\bar{\tau}}\right\} \text {, }
$$

and

$$
\Omega^{\bar{\tau}}=\{x \in \Omega:|\bar{d}(x)|>\bar{\tau}\} .
$$

Reciprocally, if (3.1) and (4.9) hold, then (4.6)-(4.7) are fulfilled.

Proof. (1) Let us prove that (4.6)-(4.7) imply (4.9). Let us argue by contradiction and suppose that (4.9) is not satisfied. Then for every $\tau^{\prime}>0$ there exists $h_{\tau^{\prime}} \in C_{\bar{u}}^{\tau^{\prime}}$ such that $\left\|h_{\tau^{\prime}}\right\|_{L^{2}(\Omega)}=1$ and

$$
\frac{\partial^{2} \mathcal{L}}{\partial u^{2}}(\bar{u}, \bar{\lambda}) h_{\tau^{\prime}}^{2}<\tau^{\prime}
$$


Since $\left\{h_{\tau^{\prime}}\right\}$ is bounded in $L^{2}(\Omega)$, there exists a subsequence, denoted in the same way, such that $h_{\tau^{\prime}} \rightarrow h$ weakly in $L^{2}(\Omega)$. We have that $h \in C_{\bar{u}}^{0}$. Indeed relations (4.3) are obtained for $h$ by passing to the limit in the corresponding ones satisfied by $h_{\tau^{\prime}}$. Let us see that $h(x)=0$ in $\Omega^{0}$

$$
\begin{aligned}
\int_{\Omega}|h(x)||\bar{d}(x)| \mathrm{d} x & =\int_{\Omega} h(x) \bar{d}(x) \mathrm{d} x \\
& =\lim _{\tau^{\prime} \rightarrow 0} \int_{\Omega} h_{\tau^{\prime}}(x) \bar{d}(x) \mathrm{d} x=\lim _{\tau^{\prime} \rightarrow 0} \int_{\Omega \backslash \Omega^{\tau^{\prime}}}\left|h_{\tau^{\prime}}(x) \| \bar{d}(x)\right| \mathrm{d} x \\
& \leq \lim _{\tau^{\prime} \rightarrow 0} \tau^{\prime} \int_{\Omega}\left|h_{\tau^{\prime}}(x)\right| \mathrm{d} x \leq \lim _{\tau^{\prime} \rightarrow 0} \tau^{\prime} \sqrt{m(\Omega)}\left\|h_{\tau^{\prime}}\right\|_{L^{2}(\Omega)}=0 .
\end{aligned}
$$

Hence $h(x) \bar{d}(x)=0$, therefore $h(x)=0$ for a.e. $x \in \Omega^{0}$ and then $h \in C_{\bar{u}}^{0}$.

Inequality (4.6) can be written in the way $\bar{H}_{u u}(x) \geq \mu>0$ in $\Omega \backslash \Omega^{\tau}$. Since $\Omega^{\tau} \subset \Omega^{\tau^{\prime}} \subset \Omega^{0}$ for $0<\tau^{\prime}<\tau$, $h_{\tau^{\prime}}=0$ in $\Omega^{\tau^{\prime}}$ and $h=0$ in $\Omega^{0}$, we have that

$$
\begin{aligned}
\liminf _{\tau^{\prime} \rightarrow 0} \int_{\Omega} \bar{H}_{u u}(x) h_{\tau^{\prime}}^{2}(x) \mathrm{d} x & =\liminf _{\tau^{\prime} \rightarrow 0} \int_{\Omega \backslash \Omega^{\tau}} \bar{H}_{u u}(x) h_{\tau^{\prime}}^{2}(x) \mathrm{d} x \\
& \geq \int_{\Omega \backslash \Omega^{\tau}} \bar{H}_{u u}(x) h^{2}(x) \mathrm{d} x=\int_{\Omega} \bar{H}_{u u}(x) h^{2}(x) \mathrm{d} x .
\end{aligned}
$$

Therefore, using the definition of $h_{\tau^{\prime}}$ along with the strong convergence $z_{h_{\tau^{\prime}}} \rightarrow z_{h}$ in $H^{1}(\Omega) \cap C(\bar{\Omega})$, we get

$$
\begin{aligned}
0 & \geq \limsup _{\tau^{\prime} \rightarrow 0} \frac{\partial^{2} \mathcal{L}}{\partial u^{2}}(\bar{u}, \bar{\lambda}) h_{\tau^{\prime}}^{2} \geq \liminf _{\tau^{\prime} \rightarrow 0} \frac{\partial^{2} \mathcal{L}}{\partial u^{2}}(\bar{u}, \bar{\lambda}) h_{\tau^{\prime}}^{2} \\
& =\liminf _{\tau^{\prime} \rightarrow 0}\left\{\int_{\Omega} \bar{H}_{u u}(x) h_{\tau^{\prime}}^{2}(x) \mathrm{d} x+\int_{\Omega} \bar{H}_{y y}(x) z_{h_{\tau^{\prime}}}^{2}(x) \mathrm{d} x+2 \int_{\Omega} \bar{H}_{y u}(x) h_{\tau^{\prime}}(x) z_{\tau_{\tau^{\prime}}}(x) \mathrm{d} x\right\} \geq \frac{\partial^{2} \mathcal{L}}{\partial u^{2}}(\bar{u}, \bar{\lambda}) h^{2},
\end{aligned}
$$

which, together with (4.7), implies that $h=0$. Finally, using the weak convergence $h_{\tau^{\prime}} \rightarrow 0$ in $L^{2}(\Omega)$ and the strong convergence $z_{h_{\tau^{\prime}}} \rightarrow 0$ in $H^{1}(\Omega) \cap C(\bar{\Omega})$, we conclude that

$$
\begin{aligned}
\mu & =\mu \limsup _{\tau^{\prime} \rightarrow 0}\left\|h_{\tau^{\prime}}\right\|_{L^{2}(\Omega)}^{2} \leq \limsup _{\tau^{\prime} \rightarrow 0} \int_{\Omega} \bar{H}_{u u}(x) h_{\tau^{\prime}}^{2}(x) \mathrm{d} x \\
& \leq \limsup _{\tau^{\prime} \rightarrow 0}\left\{\frac{\partial^{2} \mathcal{L}}{\partial u^{2}}(\bar{u}, \bar{\lambda}) h_{\tau^{\prime}}^{2} \mathrm{~d} x-\int_{\Omega} \bar{H}_{y y}(x) z_{h_{\tau^{\prime}}}^{2}(x) \mathrm{d} x-2 \int_{\Omega} \bar{H}_{y u}(x) h_{\tau^{\prime}}(x) z_{h_{\tau^{\prime}}}(x) \mathrm{d} x\right\} \leq 0 .
\end{aligned}
$$

Thus we have the contradiction.

(2) Let us prove that (4.9) implies (4.6)-(4.7). Since $C_{\bar{u}}^{0} \subset C_{\bar{u}}^{\bar{\tau}}$ for any $\bar{\tau}>0$, it is clear that (4.9) implies (4.7). Let us prove (4.6). Relation (4.8) implies that $\bar{u}$ is a local solution of problem

$$
\left(\mathrm{P}_{\delta}\right)\left\{\begin{array}{l}
\min J_{\delta}(u)=J(u)-\frac{\delta}{2}\|u-\bar{u}\|_{L^{2}(\Omega)}^{2} \\
\text { subject to }\left(y_{u}, u\right) \in\left(C(\bar{\Omega}) \cap H^{1}(\Omega)\right) \times L^{\infty}(\Omega), \\
\alpha(x) \leq u(x) \leq \beta(x) \quad \text { a.e. } x \in \Omega, \\
y_{u}\left(x_{j}\right)=\sigma_{j}, \quad 1 \leq j \leq n_{e}, \\
y_{u}\left(x_{j}\right) \leq \sigma_{j}, \quad n_{e}+1 \leq j \leq n_{e}+n_{i} .
\end{array}\right.
$$


Since $\bar{u}$ satisfies (3.1) and $J^{\prime}(\bar{u})=J_{\delta}^{\prime}(\bar{u})$, then the first order optimality conditions (3.2)-(3.3) hold. Moreover we can apply Theorem 4.1 to this problem and deduce

$$
\begin{aligned}
& \frac{\partial^{2} L}{\partial u^{2}}(x, \bar{y}(x), \bar{u}(x))-\delta \geq 0 \quad \text { a.e. } x \in \Omega \backslash \Omega^{0} \\
& \frac{\partial^{2} \mathcal{L}}{\partial u^{2}}(\bar{u}, \bar{\lambda}) h^{2}-\delta\|h\|_{L^{2}(\Omega)}^{2} \geq 0 \quad \forall h \in C_{\bar{u}}^{0} .
\end{aligned}
$$

Obviously inequality (4.11) implies (4.7). Let us prove (4.6). In the case where the bound constraints on the control are not active at all, i.e. $\alpha(x)<\bar{u}(x)<\beta(x)$ a.e., we have that $\bar{d}(x)=0$ a.e. in $\Omega$ and the Lebesgue measure of $\Omega^{0}$ is zero; hence (4.10) implies (4.6). Let us analyze the case where $\Omega^{0}$ has a strictly positive Lebesgue measure. We will proceed by contradiction and we assume that there exist no $\mu>0$ and $\tau>0$ such that (4.6) is satisfied. Then we define for every integer $k \geq 1 / \bar{\tau}$

$$
\hat{h}_{k}(x)=\left\{\begin{array}{cl}
+1 & \text { if } 0<|\bar{d}(x)| \leq 1 / k, \bar{H}_{u u}(x)<1 / k, \text { and } \bar{u}(x)=\alpha(x), \\
-1 & \text { if } 0<|\bar{d}(x)| \leq 1 / k, \bar{H}_{u u}(x)<1 / k, \text { and } \bar{u}(x)=\beta(x), \\
0 & \text { otherwise }
\end{array}\right.
$$

Since (4.6) is not satisfied for $\mu=1 / k$ and $\tau=1 / k$, with arbitrarily large $k$, and (4.10) implies that $\bar{H}_{u u}(x) \geq \delta$ if $\bar{d}(x)=0$, we have that $\hat{h}_{k} \neq 0$. Then we define $\tilde{h}_{k}=\hat{h}_{k} /\left\|\hat{h}_{k}\right\|_{L^{2}(\Omega)}$. Let us prove that $\tilde{h}_{k} \rightarrow 0$ weakly in $L^{2}(\Omega)$. Taking into account that $\operatorname{supp}\left\{\tilde{h}_{k}\right\} \subset \operatorname{supp}\left\{\tilde{h}_{k^{\prime}}\right\}$ for every $k>k^{\prime}$ and $\cap_{k \geq \frac{1}{\tau}} \operatorname{supp}\left\{\tilde{h}_{k}\right\}=\emptyset$, we deduce that $\tilde{h}_{k}(x) \rightarrow 0$ pointwise a.e. in $\Omega$. On the other hand, $\left\{\tilde{h}_{k}\right\}_{k=1}^{\infty}$ is bounded in $L^{2}(\Omega)$; consequently $\tilde{h}_{k} \rightarrow 0$ weakly in $L^{2}(\Omega)$; see Hewitt and Stromberg [13], p. 207. Furthermore we have that $\tilde{h}_{k}(x)=0$ if $|\bar{d}(x)|>1 / k$ and $\tilde{h}_{k}$ satisfies the last sign conditions of (4.3). Let us define a new function $h_{k} \in C_{\bar{u}}^{\bar{\tau}}$ close to $\tilde{h}_{k}$. Using the functions $\left\{\bar{w}_{j}\right\}_{j \in I_{0}}$ introduced in (3.1), we set

$$
h_{k}=\tilde{h}_{k}-\sum_{j \in I_{0}} \alpha_{k j} \bar{w}_{j}, \quad \text { with } \alpha_{k j}=G_{j}^{\prime}(\bar{u}) \tilde{h}_{k} .
$$

As in the proof of Theorem 4.1, we deduce that $h_{k}$ satisfies (4.3) and $h_{k}(x)=0$ if $|\bar{d}(x)|>1 / k \geq \bar{\tau}$, hence $h_{k} \in C_{\bar{u}}^{\bar{\tau}}$. Moreover, since $\tilde{h}_{k} \rightarrow 0$ weakly in $L^{2}(\Omega)$, we deduce that $\alpha_{k j} \rightarrow 0$, and therefore $h_{k} \rightarrow 0$ weakly in $L^{2}(\Omega)$ too. On the other hand, since $\operatorname{supp}\left\{\tilde{h}_{k}\right\}$ is included in the set of points of $\Omega$ where the bound constraints on the control are active, which has an empty intersection with the support of each function $\bar{w}_{j}\left(j \in I_{0}\right)$, it follows

$$
\begin{aligned}
\left\|h_{k}\right\|_{L^{2}(\Omega)} & =\left\{\int_{\operatorname{supp}\left\{\tilde{h}_{k}\right\}} h_{k}^{2} \mathrm{~d} x+\int_{\Omega \backslash \operatorname{supp}\left\{\tilde{h}_{k}\right\}} h_{k}^{2} \mathrm{~d} x\right\}^{\frac{1}{2}} \\
& =\left\{\int_{\operatorname{supp}\left\{\tilde{h}_{k}\right\}} \tilde{h}_{k}^{2} \mathrm{~d} x+\int_{\Omega \backslash \operatorname{supp}\left\{\tilde{h}_{k}\right\}}\left(\sum_{j \in I_{0}} \alpha_{k j} \bar{w}_{j}\right)^{2} \mathrm{~d} x\right\}^{\frac{1}{2}} \\
& =\left\{\left\|\tilde{h}_{k}\right\|_{L^{2}(\Omega)}^{2}+\left\|\sum_{j \in I_{0}} \alpha_{k j} \bar{w}_{j}\right\|_{L^{2}(\Omega)}^{2}\right\} \\
& \geq\left\{1-2 \sum_{j \in I_{0}} \alpha_{k j}^{2}\left\|\bar{w}_{j}\right\|_{L^{2}(\Omega)}^{2}\right\}^{\frac{1}{2}} \stackrel{k \rightarrow \infty}{\longrightarrow} 1 .
\end{aligned}
$$


From this relation and (4.9) with $h=h_{k}$, we get

$$
\delta \leq \delta \liminf _{k \rightarrow \infty}\left\|h_{k}\right\|_{L^{2}(\Omega)}^{2} \leq \liminf _{k \rightarrow \infty} \frac{\partial^{2} \mathcal{L}}{\partial u^{2}}(\bar{u}, \bar{\lambda}) h_{k}^{2} .
$$

On the other hand, the weak convergence $h_{k} \rightarrow 0$ in $L^{2}(\Omega)$ implies the strong convergence $z_{h_{k}} \rightarrow 0$ in $H^{1}(\Omega) \cap C(\bar{\Omega})$. Now taking into account that $\bar{H}_{u u}(x)<1 / k$ in the support of $\tilde{h}_{k}$ and $\alpha_{k j} \rightarrow 0$, we get

$$
\begin{aligned}
\liminf _{k \rightarrow \infty} \frac{\partial^{2} \mathcal{L}}{\partial u^{2}}(\bar{u}, \bar{\lambda}) h_{k}^{2} & \leq \limsup _{k \rightarrow \infty} \int_{\Omega} \bar{H}_{u u}(x) h_{k}^{2}(x) \mathrm{d} x+\limsup _{k \rightarrow \infty} \int_{\Omega} \bar{H}_{y y}(x) z_{h_{k}}^{2}(x) \mathrm{d} x \\
& +2 \limsup _{k \rightarrow \infty} \int_{\Omega} \bar{H}_{y u}(x) h_{k}(x) z_{h_{k}}(x) \mathrm{d} x \leq \limsup _{k \rightarrow \infty} \int_{\operatorname{supp}\left\{\tilde{h}_{k}\right\}} \bar{H}_{u u}(x) h_{k}^{2}(x) \mathrm{d} x \\
& +\limsup _{k \rightarrow \infty} \int_{\Omega \backslash \operatorname{supp}\left\{\tilde{h}_{k}\right\}} \bar{H}_{u u}(x) h_{k}^{2}(x) \mathrm{d} x \leq \limsup _{k \rightarrow \infty} \frac{1}{k} \int_{\operatorname{supp}\left\{\tilde{h}_{k}\right\}} \tilde{h}_{k}^{2}(x) \mathrm{d} x \\
& +\limsup _{k \rightarrow \infty} \int_{\Omega \backslash \operatorname{supp}\left\{\tilde{h}_{k}\right\}} \bar{H}_{u u}(x)\left[\sum_{j \in I_{0}} \alpha_{k j} \bar{w}_{j}(x)\right]^{2} \mathrm{~d} x=\limsup _{k \rightarrow \infty} \frac{1}{k} \int_{\Omega} \tilde{h}_{k}^{2}(x) \mathrm{d} x=\lim _{k \rightarrow \infty} \frac{1}{k}=0,
\end{aligned}
$$

which contradicts (4.12).

\section{REFERENCES}

[1] N. Arada, E. Casas and F. Tröltzsch, Error estimates for the numerical approximation of a semilinear elliptic control problem. Comput. Optim. Appl. 23 (2002) 201-229.

[2] J.F. Bonnans and E. Casas, Contrôle de systèmes elliptiques semilinéaires comportant des contraintes sur l'état, in Nonlinear Partial Differential Equations and Their Applications, Collège de France Seminar 8, H. Brezis and J.-L. Lions Eds., Longman Scientific \& Technical, New York (1988) 69-86.

[3] E. Casas, Pontryagin's principle for optimal control problems governed by semilinear elliptic equations, in International Conference on Control and Estimation of Distributed Parameter Systems: Nonlinear Phenomena, F. Kappel and K. Kunisch Eds., Basel, Birkhäuser, Int. Series Num. Analysis. 118 (1994) 97-114.

[4] E. Casas, Error estimates for the numerical approximation of semilinear elliptic control problems with finitely many state constraints. ESAIM: COCV 8 (2002) 345-374.

[5] E. Casas and M. Mateos, Second order optimality conditions for semilinear elliptic control problems with finitely many state constraints. SIAM J. Control Optim. 40 (2002) 1431-1454.

[6] E. Casas and M. Mateos, Uniform convergence of the FEM. Applications to state constrained control problems. Comp. Appl. Math. 21 (2002) 67-100.

[7] E. Casas and F. Tröltzsch, Second order necessary optimality conditions for some state-constrained control problems of semilinear elliptic equations. App. Math. Optim. 39 (1999) 211-227.

[8] E. Casas and F. Tröltzsch, Second order necessary and sufficient optimality conditions for optimization problems and applications to control theory. SIAM J. Optim. 13 (2002) 406-431.

[9] E. Casas, J.P. Raymond and H. Zidani, Optimal control problems governed by semilinear elliptic equations with integral control constraints and pointwise state constraints, in International Conference on Control and Estimations of Distributed Parameter Systems, W. Desch, F. Kappel and K. Kunisch Eds., Basel, Birkhäuser, Int. Series Num. Analysis. 126 (1998) $89-102$.

[10] E. Casas, F. Tröltzsch and A. Unger, Second order sufficient optimality conditions for some state constrained control problems of semilinear elliptic equations. SIAM J. Control Optim. 38 (2000) 1369-1391.

[11] F.H. Clarke, A new approach to Lagrange multipliers. Math. Op. Res. 1 (1976) 165-174.

[12] P. Grisvard, Elliptic Problems in Nonsmooth Domains. Pitman, Boston-London-Melbourne (1985).

[13] E. Hewitt and K. Stromberg, Real and abstract analysis. Springer-Verlag, Berlin-Heidelberg-New York (1965).

[14] W. Littman and G. Stampacchia and H.F. Weinberger, Regular points for elliptic equations with discontinuous coefficients. Ann. Scuola Normale Sup. Pisa 17 (1963) 43-77.

[15] M. Mateos, Problemas de control óptimo gobernados por ecuaciones semilineales con restricciones de tipo integral sobre el gradiente del estado. Ph.D. thesis, University of Cantabria, Spain (2000). 
[16] H. Maurer and J. Zowe, First- and second-order conditions in infinite-dimensional programming problems. Math. Program. 16 (2000) 431-450.

[17] J.-P. Raymond and F. Tröltzsch, Second order sufficient optimality conditions for nonlinear parabolic control problems with state constraints. Discrete Contin. Dynam. Systems 6 (1979) 98-110.

[18] S.M. Robinson, Stability theory for systems of inequalities, Part II: Differentiable nonlinear systems. SIAM J. Numer. Anal. 13 (1976) 497-513.

[19] J.C. Saut and B. Scheurer, Sur l'unicité du problème de Cauchy et le prolongement unique pour des équations elliptiques à coefficients non localement bornés. J. Differential Equations 43 (1982) 28-43. 